\title{
Low-Level Laser Therapy in the Treatment of Inferior Alveolar Nerve Paresthesia After Surgical Exeresis of a Complex Odontoma
}

\author{
Ícaro Girão Evangelista ${ }^{1}$, Fernando Bruno Pontes Tabosa ${ }^{1}$, Ariel Valente Bezerra ${ }^{2}$, Eliziário Vitoriano de Araújo \\ Neto $\mathrm{Jr}^{2^{*}}$ \\ ${ }^{1}$ Undergraduate in Dentistry, University of Fortaleza, Fortaleza, Ceara, Brazil \\ ${ }^{2}$ Department of Oral and Maxillofacial Surgery, Baptist Memorial Hospital, Fortaleza, Ceara, Brazil
}

\author{
*Correspondence to \\ Eliziário Vitoriano de Araújo Neto Jr, \\ Rua Professor Dias da Rocha, 1530 \\ - Aldeota, Fortaleza (CE), Ceará, \\ Brazil. Zip Code: 60170-311; Phone: \\ + 55853087.7831 \\ Email: eliziariojunior@gmail.com \\ Published online October 1, 2019
}

\begin{abstract}
Introduction: Orofacial paresthesia is due to trauma to the neural structure of a particular nerve. In dentistry alterations caused by nerve damage in most cases are presented with transient symptomatology. However, it has been agreed by several authors that persistent inferior alveolar sensory aberrations for more than 6 months leave some degree of disability or are considered permanent. The objective of the present study is to report the clinical case of a young patient submitted to low-level laser therapy for the treatment of paresthesia of the inferior alveolar nerve after removal of a complex odontoma in the posterior region of the mandible.

Methods: Twenty-four hours after the surgical procedure the patient started the low-level laser therapy with the following parameters: $100 \mathrm{~mW}$ of potency, $140 \mathrm{~J} / \mathrm{cm}^{2}$ of energy density, $4 \mathrm{~J}$ of energy per application point, 40 seconds of application per point and $0.028 \mathrm{~cm}^{2}$ of spot area. For this particular case, the technique of alternation of laser wavelengths was used, in the first session of which visible red of $660 \mathrm{~nm}$ was applied, followed by near-infrared of $808 \mathrm{~nm}$ and so on.

Results: In the first session, the score on the visual analog scale (VAS) was " 3 ". In the tenth and last sessions, the patient reported a VAS " 9 ".

Conclusions: It seems that the early initiation of the low-level laser therapy favors a better outcome in cases like the one presented in this paper. The technique of alternation of laser wavelengths between sessions seems to have some role in the outcome possibly because of the constant stimulation of different chromophores along the treatment course. These two factors need further confirmation and validation through randomized clinical trials.

Keywords: Lasers; Low-Level Light Therapy; Paresthesia.
\end{abstract}

\section{Introduction}

It is widely known within the universe of dentistry that paresthesia occurs in several dental procedures such as the removal of mandibular third molars near the mandibular canal, which eventually causes irreversible damage to the nervous structure and maxillofacial traumas, leading to dysfunction in the nerve and orthognathic surgery in the prognathism or mandibular retrognathism. ${ }^{1}$ There are also cases of paresthesia in endodontic treatment ${ }^{2}$ and implantology. ${ }^{3}$ The occurrence of paresthesia after the removal of pathological lesions of large proportion is often inevitable. ${ }^{4}$

Low-level laser therapy has several therapeutic effects reported in the literature, such as acceleration of wound healing, reduction of pain, trismus and edema, ${ }^{5}$ stimulation of endorphin release, modulation of the immune system ${ }^{6}$ and neural repair, allowing for possible objective and subjective improvements in sensory perception in a short-term and long-term postoperative period.

Therefore, the objective of the present study was to report the clinical case of a young patient submitted to low-level laser therapy for the treatment of paresthesia of the inferior alveolar nerve after the removal of a complex odontoma in the posterior region of the mandible.

\section{Case Presentation}

A 22-year-old healthy white man was referred to the outpatient clinic of the Department of Oral and Maxillofacial Surgery for the evaluation of a volume increase in his oral cavity.

At the extra-oral physical examination, a discrete

Please cite this article as follows: Girão Evangelista IG, Pontes Tabosa FB, Bezerra AV, de Araújo Neto EV Jr. Low-level laser therapy in the treatment of inferior alveolar nerve paresthesia after surgical exeresis of a complex odontoma. J Lasers Med Sci. 2019;10(4):342-345. doi:10.15171/jlms.2019.55. 
volume increase was observed in the mandible angle of the right side, firm to palpation and painless. In the intraoral physical examination, it was also possible to observe a volume increase in the region of the retromolar trigone and buccal cortical of the mandibular angle.

In the panoramic radiographic examination, it was possible to verify the radiopaque lesion, well delimited, solid aspect, occupying the region of the mandibular angle, posterior to the first right lower molar (Figure 1). It was also possible to observe the impacted second molar at the base of the mandible, with severe root laceration and agenesis of the third molar. At this point, it was established as a diagnostic hypothesis of a complex odontoma. Figure 2 shows the radiographic aspect of 30 days after surgery.

After performing the lesion excision procedure, the surgical specimen was sent for histopathological examination wherein the diagnostic hypothesis of the complex odontoma was confirmed. Given the proximity of the lesion to the inferior alveolar nerve, the surgeon responsible for the treatment wrote down in the patient's chart that there was direct neural trauma and the patient invariably would evolve toward orofacial paresthesia of the inferior alveolar nerve. The low-level laser therapy was planned to begin 24 hours after the surgical procedure.

To perform the low-level laser therapy in this case, we used the following parameters: $100 \mathrm{~mW}$ of potency, $140 \mathrm{~J} /$ $\mathrm{cm}^{2}$ of energy density, $4 \mathrm{~J}$ of energy per application point, 40 seconds of application per point and $0.028 \mathrm{~cm}^{2}$ of spot area. We used the technique of alternation of wavelengths; that is, in one session visible red of $660 \mathrm{~nm}$ was made and the other with infrared of $808 \mathrm{~nm}$ and so on respectively. The application points totaling 52 were defined as follows: 10 extra-oral points in the inferior alveolar nerve path (Figure 3), 16 extra-oral points in the mental region (Figure 4), 16 intra-oral points in the lower labial mucosa and 10 intra-oral points beginning in the vestibular fornix in the central incisor region to the retromolar region.

In order to follow the evolution of the treatment, a visual analog scale (VAS) was established from " 0 " to " 10 " representing a lack of sensitivity and sensitivity within normal levels respectively.

At the first consultation with the patient, little sensitivity was indicated in the mental region, lower lip, labial mucosa, and posterior region of the mandible on the surgery side. On this occasion, he marked a VAS 3.

After the first low-level laser therapy session, the patient reported feeling a slight tingling in the mental region. In the fourth session, already reporting a VAS 5 , he reported improvement on drinking water as well as discerning when the liquid ran down through the labial commissure. At the end of the eighth session, with a VAS 7, the patient reported that he was satisfied with the overall improvement as a result of low-level laser therapy.

Two months after the tenth and last sessions of lowlevel laser therapy, a return visit with the patient was conducted. When asked about the VAS sensitivity, he

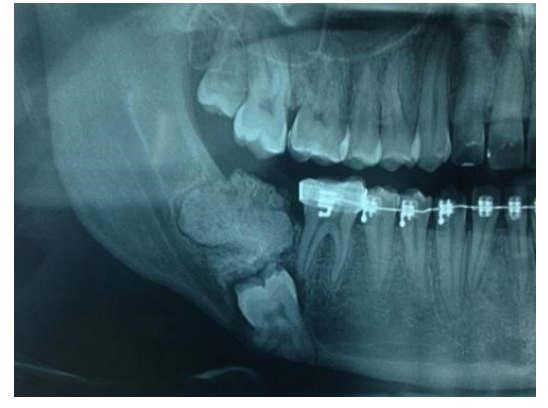

Figure 1. Radiographic Aspect of the Lesion in the Posterior Aspect of the Mandible.

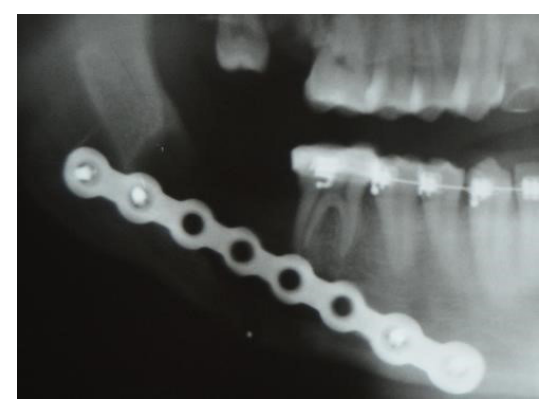

Figure 2. Postoperative Radiographic Aspect of 30 Days After Surgery.

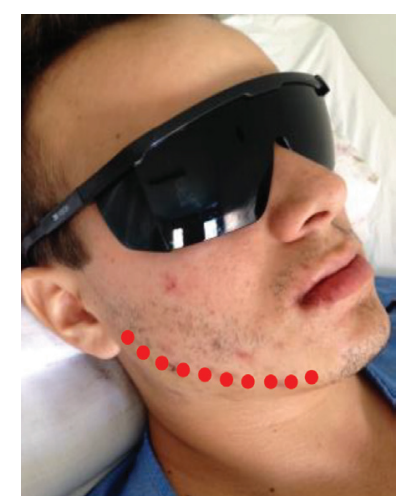

Figure 3. 10 Extra-oral Points in the Inferior Alveolar Nerve Path.

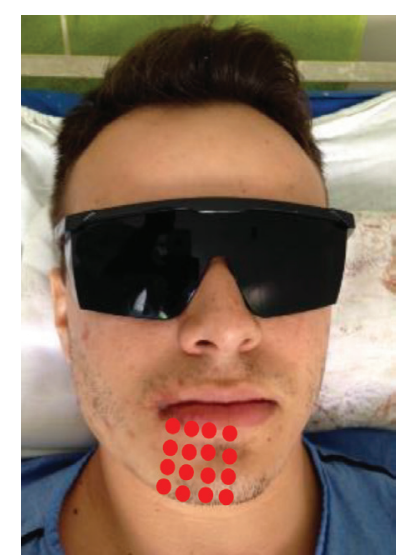

Figure 4. 6 Extra-oral Points in the Mental Region.. 
indicated a 9, representing an improvement of two scores compared to the last session. At the 2-year follow-up, the patient did not report significant improvements, reporting that he still felt a slight difference in sensitivity when draining pimples on the right side of the mental region, for example. However, in the general aspect of the treatment, he reported being satisfied with the gain in sensitivity obtained during treatment with low-level laser therapy.

The information obtained for this case report was authorized by the patient through a free and informed consent form attached to his clinical chart.

\section{Discussion}

Low-level laser therapy has been the subject of previous studies in order to find out the size of its participation in the repair of neural lesions. A study of 13 patients after the use of low-level laser therapy found improvements in mechanical sensory perception. ${ }^{7}$ The individuals targeted by this research showed a permanent reduction of sensitivity for two years, caused by mandibular sagittal osteotomy, and consequently, compression or traction of the inferior alveolar nerve.

Coinciding with our result, Miloro and Repasky also observed improvement in 6 patients submitted to mandibular sagittal osteotomy. ${ }^{1}$ In this study, seven lowlevel laser therapy sessions were performed along the inferior alveolar nerve distribution, immediately before surgery, 6 and 24 hours after surgery and on the second, third, fourth and seventh post-operative days. As a result, an increase in the sensorineural response was observed, with a return to normal within two months. For this study, the following low-level laser therapy parameters were used: $70 \mathrm{~mW}$ of potency, $820 \mathrm{~nm}$ of wavelength (infrared), $0.13 \mathrm{~cm}^{2}$ of spot area, $550 \mathrm{~mW} / \mathrm{cm}^{2}$ of power density and 6J of energy per point of application.

Some authors claim that no conclusion can be drawn about whether there is any method that provides beneficial results in neural repair. One study ${ }^{8}$ cited several possible techniques for treating neural lesions, such as surgical therapies like internal and external neurolysis, neurorrhaphy, autogenous nerve transplantation, and non-surgical therapies, including low-level laser therapy and drug therapy. The conclusion reached by the authors is that these techniques have not yet been fully validated in randomized clinical trials and that there is also a lack of evidence comparing these methods or approaching the ideal time to perform the procedure.

We conclude that despite the obvious open field that is low-level laser therapy for the treatment of orofacial paresthesia and its apparent success published in some studies in the literature, it is still necessary randomized long term clinical studies that can demonstrate a true efficacy of this promising non-surgical treatment modality. The evaluation of the results obtained from low-level laser therapy for the treatment of orofacial paresthesia is still mainly in the subjective field, which may represent an important slant of evaluation, since pain threshold between humans can vary appreciably. More objective methods should be investigated to assure greater reliability in the follow-up cases similar to the one presented in this article.

It seems that the early initiation of the low-level laser therapy produces a better outcome in cases like the one presented in this paper. The technique of alternation of laser wavelengths between sessions seems to have some role in the outcome possibly because of the constant stimulation of different chromophores along the treatment course. These two factors need further confirmation and validation through randomized clinical trials.

\section{Ethical Considerations}

An informed consent was obtained from the patient for publication of this report.

\section{Conflict of Interests}

The authors declare no conflict of interest.

\section{Acknowledgment}

Acknowledgments to Baptist Memorial Hospital for clinical support.

\section{References}

1. MiloroM,RepaskyM.Low-levellaser effecton neurosensory recovery after sagittal ramus osteotomy. Oral Surg Oral Med Oral Pathol Oral Radiol Endod.2000;89(1):12-8. doi:10.1016/S1079-2104(00)80006-2.

2. Jerjes W, Swinson B, Banu B, Al Khawalde M, Hopper C. Paraesthesia of the lip and chin area resolved by endodontic treatment: A case report and review of literature. Br Dent J. 2005, 198:743-745. doi:10.1038/sj.bdj.4812412.

3. Kraut RA, Chahal O: Management of patients with trigeminal nerve injuries after mandibular implant placement. J Am Dent Assoc. 2002;133(10):1351-4. doi: 10.14219/jada.archive.2002.0050.

4. Aziz SR, Pulse C, Dourmas MA, Roser SM: Inferior alveolar nerve paresthesia associated with a mandibular dentigerous cyst. J Oral Maxillofac Surg. 2002;60(4):457-9. doi: 10.1053/joms.2002.31238.

5. Ferrante M, Petrini M, Trentini P, Perfetti G, Spoto G. Effect of low-level laser therapy after extraction of impacted lower third molars. Lasers Med Sci. 2013;28(3):845-9. doi: 10.1007/s10103-012-1174-4.

6. Hagiwara S, Iwasaka H, Okuda K, Noguchi T. GaAlAs $(830 \mathrm{~nm})$ low-level laser enhances peripheral endogenous opioid analgesia in rats. Lasers Surg Med. 2007;39(10):797802.doi: 10.1002/lsm.20583.

7. Khullar SM, Brodin P, Barkvoll P, Haanæs HR. Preliminary study of low-level laser for treatment of long-standing sensory aberrations in the inferior alveolar nerve. J Oral Maxillofac Surg. 1996;54(1):2-7. doi:10.1016/s02782391(96)90290-6.

8. Renton TF, Coulthard P, Esposito M. Interventions for iatrogenic inferior alveolar nerve injury. Cochrane Database Syst Rev. 2005(2). doi: 10.1002/14651858.CD005293. 